\title{
Roles of the Ratio of C-Reactive Protein to Serum and Pericardial Fluid Albumin Levels in Predicting in-Hospital Mortality in Patients Undergoing Pericardiocentesis
}

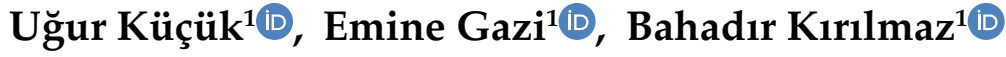 \\ 1 Onsekiz Mart University Faculty of Medicine, Department of Cardiology, Çanakkale, Turkey
}

\begin{abstract}
Background: Pericardial effusions occur due to excessive fluid accumulation in the pericardial space. In effusions not responding to medical treatment, pericardiocentesis is an important method of treatment affecting prognosis. CRP/albumin ratios have been found to be associated with prognosis in conditions such as cardiac failure, sepsis, malignancy, and the routinely available parameters used for the prognosis prediction of the patients who underwent pericardiocentesis are limited. This study aimed to examine the usability of CRP/albumin and CRP/pericardial fluid albumin (CRP/pf-albumin) ratios as predictors for in-hospital mortality of patients who underwent surgery pericardiocentesis.
\end{abstract}

Methods: This study included 54 patients (25 females and 29 males). All patients underwent pericardiocentesis.

Results: The average age was $67 \pm 14$ years. When the groups were compared with each other, CRP, CRP/albumin ratio and CRP/ pf-Albumin ratio were higher in the in-hospital mortality group compared to the group discharged with recovery [19 (14-25), 6.3 (1-30), $\mathrm{p}<0.001 ; 4.27$ (3.87-12.02), 1.9 (0.24-10.38), $\mathrm{p}<0.001 ; 7$ (6.25-13.59), 2.5 (0.28-12.22), $\mathrm{p}<0.001$, respectively]. In the univariate logistic regression analysis, CRP (odds ratio [OR]: 0.821, P: 0.004, 95.0\% confidence interval [CI]: 0.918-1.049), CRP/albumin ratio (OR: 0.600, P: 0.011, 95.0\% CI: 0.406-0.888) and CRP/pf-Albumin ratio (OR: 0.608, P: 0.004, 95.0\% CI: 0.431-0.856) was found to be associated with in-hospital mortality in patients who underwent pericardiocentesis.

Conclusion: For the first time in the literature, we demonstrated that CRP/albumin and CRP/pf-Albumin ratios are associated with in-hospital mortalities in patients who underwent pericardiocentesis, irrespective of the etiology.

Keywords: C-Reactive Protein/ Albumin Ratio, Pericardial Fluid Albumin, Mortality, Hospitalized Patients. 


\section{INTRODUCTION}

Pericardial fluid is the serous fluid released from the pericardium surrounding the heart to the pericardial space. It helps the heart function more efficiently by holding it in place within the chest cavity and preventing it from rubbing against other tissues. The pericardial sac contains approximately $10-50 \mathrm{~mL}$ fluid, and the increased secretion or impaired reabsorption of the fluid results in moderate to severe effusions. While the mild fluid accumulations respond to medical treatment, severe fluid accumulations lead to cardiac dysfunction, and pericardiocentesis is the most beneficial therapeutic procedure unless clinically contraindicated. ${ }^{1,2}$

Pericardial effusions cause clinical signs in patients based on their onset type and size. While they may be asymptomatic, fatal clinical presentations may also be observed. Viral, bacterial, neoplastic, autoimmune, and iatrogenic causes are the most common reasons in the clinical practice; the underlying etiology and the size of the fluid accumulation is associated with the prognosis, and there are no clear biochemical parameters recommended by the guidelines, which can be used for in-hospital mortality. ${ }^{3}$

Being functional in many clinical conditions, albumin and $\mathrm{C}$-reactive protein (CRP) can provide information on inflammatory processes. Several studies have shown that increased CRP levels provide information on prognosis in conditions such as malignancy, stroke, coronary artery disease (CAD), and heart failure (HF). ${ }^{4-}$ ${ }^{6}$ Similarly, albumin levels have been documented to provide information on inflammatory conditions. ${ }^{7}$ In another study, the CRP-to-albumin ratio was shown to be an independent risk factor for mortality in patients with traumatic brain injury (TBI). ${ }^{8}$

On the contrary, it is not clear whether albumin ratios in the pericardial fluid can be used to predict the patients who underwent pericardiocentesis. Using a combination of parameters rather than individual parameters may provide more information.

Our study aimed to examine the usability of CRP-toalbumin and CRP-to-pericardial fluid albumin ratios in predicting in-hospital mortality in patients who underwent pericardiocentesis.

\section{MATERIALS AND METHOD}

\section{Study Population and Data Collection}

Our retrospective study enrolled 54 patients who consecutively underwent pericardiocentesis from January 2013 to December 2020 in our university hospital. Patients included in the study were those who did not respond to medical treatment with moderate-large pericardial effusion. The study consisted of patients who underwent pericardiocentesis for the first time. Patients who had to undergo surgical treatment due to pericardiocentesis failure, any contraindication of pericardiocentesis, constrictive pericarditis, and patients under 18 years of age were excluded from the study.

For biochemical parameters, blood samples taken from the upper extremity peripheral venous route and pericardial fluid samples collected during pericardiocentesis were studied within the same timeframe, and data for the variables were obtained. Hemogram, CRP, complete blood count $(\mathrm{CBC})$, pericardial fluid albumin, and lactate dehydrogenase (LDH) tests were studied for all patients. Demographic information, clinical results, biochemical and echocardiographic data of the patients were obtained from the hospital records, and all data were compared between the groups.

Patients who were discharged with recovery were placed in Group 1, and patients who died during hospitalization were placed in Group 2.

This study was approved by the Çanakkale Onsekiz Mart University ethics committee of the hospital in which it took place in line with the recommendations of the Declaration of Helsinki (Date: 09.12.2020 Decision no: 2020-14).

\section{Pericardiocentesis procedure and definitions}

Any pericardial effusion smaller than $10 \mathrm{~mm}$ is defined as minimal effusions between 10 and $20 \mathrm{~mm}$ are defined as moderate, and effusions greater than $20 \mathrm{~mm}$ are defined as large effusion. In all patients, it was performed by Seldinger technique using $6 \mathrm{~F}$ sheath at an angle of $30^{\circ}$ towards the left shoulder under fluoroscopy in a coronary angiography laboratory setting. ${ }^{9} 500 \mathrm{ml}$ fluid was discharged from all patients in the laboratory. After successful pericardiocentesis, the pigtail catheter was left in the pericardial cavity for drainage. The catheter was removed when the control echocardiography showed effusion smaller than $10 \mathrm{~mm}$. 


\section{Echocardiographic assessment}

Pre- and post-procedural echocardiographic assessments were performed using a $2.5-\mathrm{Mhz}$ probe in the Vivid 7 Pro device (GE, Norway). LVEF values were calculated using modified Simpson's rule. TTE examinations were performed in line with the imaging guidelines recommended by the US and EU associations. ${ }^{10}$

\section{Statistical analysis}

SPSS 20.0 (SPSS Inc, Chicago, IL, USA) software was used for the statistical analysis. The distribution normality of the parameters was analyzed using the KolmogorovSmirnov test with continuous variables being expressed as mean \pm standard deviation and categorical variables being expressed as percentage and number. Independent samples t-test and Mann-Whitney U test were used for the comparison of normally and non-normally distributed parameters, respectively. A chi-squared test was used for the comparison of odds ratios for categorical variables.
Univariate logistic regression analysis was used to assess the association of biochemical and demographic parameters with in-hospital mortality in patients who underwent pericardiocentesis.

Standardized beta coefficients were calculated with 95\% confidence intervals. Receiver operating characteristic (ROC) analysis was performed to determine the ability of $\mathrm{CRP} /$ albumin and CRP/pf-Albumin ratios to predict inhospital mortality. $\mathrm{P}$ values under 0.05 were considered to be statistically significant.

\section{RESULTS}

Our study consisted of 54 patients and two groups. No life-threatening complication was observed during the pericardiocentesis procedure. The mean age of the patients was $67.87 \pm 14.91$. Table 1 shows the intergroup comparisons. No statistical difference was detected between the groups in terms of $\mathrm{LDH}$, pericardial fluid lactate dehydrogenase (pf-LDH), albumin, and pfAlbumin values ( $p>0.05$ for all) (Table 1 and 2).

Table 1. Demographic, clinical and laboratory features of the patients

\begin{tabular}{|l|c|c|c|c|}
\hline Variables & $\begin{array}{c}\text { All patients } \\
(\mathrm{n}=54)\end{array}$ & $\begin{array}{c}\text { Discharged patients } \\
(\mathrm{n}=49)\end{array}$ & $\begin{array}{c}\text { In hospital death } \\
(\mathrm{n}=5)\end{array}$ & $P$ \\
\hline Age (years) & $67.87 \pm 14.91$ & $67.51 \pm 14.12$ & $71.40 \pm 23.19$ & 0.583 \\
\hline Female $\mathrm{n}(\%)$ & $25(46)$ & $24(49)$ & $1(20)$ & 0.223 \\
\hline HT n (\%) & $15(28)$ & $14(29)$ & $1(20)$ & 0.690 \\
\hline DM n (\%) & $13(20)$ & $12(25)$ & $1(20)$ & 0.827 \\
\hline LVEF $(\%)$ & $51.17 \pm 8.17$ & $51.47 \pm 7.96$ & $48.20 \pm 10.54$ & 0.399 \\
\hline Systolic blood pressure $(\mathrm{mmHg})$ & $131.11 \pm 9.37$ & $131.47 \pm 9.20$ & $127.60 \pm 11.43$ & 0.384 \\
\hline Diastolic blood pressure $(\mathrm{mmHg})$ & $75.43 \pm 9.68$ & $75.51 \pm 10.03$ & $74.60 \pm 5.68$ & 0.844 \\
\hline Heart rate (beats/min) & $90.8 \pm 85.4$ & $90.86 \pm 89.56$ & $80.4 \pm 15.3$ & 0.788 \\
\hline Laboratory values & & & & \\
\hline Glucose $(\mathrm{mg} / \mathrm{dl})$ & $118.46 \pm 35.78$ & $117.72 \pm 36.16$ & $125.68 \pm 34.68$ & 0.640 \\
\hline Creatinine $(\mathrm{mg} / \mathrm{dL})$ & $1.22 \pm 0.73$ & $1.19 \pm 0.67$ & $1.44 \pm 1.27$ & 0.487 \\
\hline Sodium $(\mathrm{mEq} / \mathrm{L})$ & $140.81 \pm 6.97$ & $141.06 \pm 7.14$ & $138.40 \pm 4.82$ & 0.421 \\
\hline Potassium $(\mathrm{mEq} / \mathrm{L})$ & $4.25 \pm 0.70$ & $4.22 \pm 0.66$ & $4.57 \pm 1.09$ & 0.301 \\
\hline TSH $(\mathrm{mU} / \mathrm{L})$ & $2.31 \pm 1.59$ & $2.30 \pm 1.62$ & $2.32 \pm 1.46$ & 0.980 \\
\hline Alanine aminotransferase $(\mathrm{U} / \mathrm{L})$ & $38.48 \pm 55.51$ & $39.68 \pm 58.11$ & $26.76 \pm 10.70$ & 0.625 \\
\hline Aspartate aminotransferase $(\mathrm{U} / \mathrm{L})$ & $33.24 \pm 31.15$ & $34.08 \pm 32.44$ & $25.00 \pm 11.57$ & 0.540 \\
\hline Calcium $(\mathrm{mg} / \mathrm{dl})$ & $9.07 \pm 0.52$ & $9.10 \pm 0.51$ & $8.74 \pm 0.57$ & 0.140 \\
\hline LDH $(\mathrm{U} / \mathrm{L})$ & $321.63 \pm 189.70$ & $317.69 \pm 187.04$ & $360.20 \pm 234.44$ & 0.638 \\
\hline LDH of Fluid $(\mathrm{U} / \mathrm{L})$ & $575.48 \pm 880.576$ & $599.57 \pm 929.832$ & $339.40 \pm 210.165$ & 0.534 \\
\hline WBC count, $\left(x 10^{3} \mu \mathrm{L}\right)$ & $9.93 \pm 5.28$ & $10.12 \pm 5.47$ & $8.04 \pm 2.55$ & 0.407 \\
\hline Hemoglobin $\mathrm{g} / \mathrm{dL}$ & $13.66 \pm 13.88$ & $13.58 \pm 14.32$ & $14.41 \pm 9.58$ & 0.901 \\
\hline Trombosit count $\left(\times 10^{3} \mu \mathrm{L}\right)$ & $276.55 \pm 140.03$ & $282.357 \pm 142.79$ & $219.700 \pm 103.26$ & 0.345 \\
\hline Neutrophil count, $\left(\times 10^{3} / \mathrm{L}\right)$ & $7.76 \pm 4.78$ & $7.79 \pm 4.89$ & $7.50 \pm 3.94$ & 0.898 \\
\hline Lymphocyte count $\left(\times 10^{3} / \mathrm{L}\right)$ & $1.38 \pm 0.93$ & $1.39 \pm 0.93$ & $1.21 \pm 1.05$ & 0.683 \\
\hline
\end{tabular}

DM: Diabetes mellitus, HT: Hypertension, LVEF: Left ventricle ejection fraction, TSH: Thyroid Stimulating Hormone, LDH: Lactate dehydrogenase, CRP: C-Reactive protein 
Table 2. C-reactive protein and albumin levels of cardiac tamponade patients

\begin{tabular}{|l|c|c|c|c|}
\hline Variables & $\begin{array}{c}\text { All patients } \\
(\mathbf{n}=54)\end{array}$ & $\begin{array}{c}\text { Discharged patients } \\
(\mathbf{n}=49)\end{array}$ & $\begin{array}{c}\text { In hospital death } \\
(\mathbf{n}=5)\end{array}$ & P \\
\hline Albumin $(\mathrm{g} / \mathrm{dl})$ & $3.58 \pm 0.52$ & $3.60 \pm 0.49$ & $3.48 \pm 0.87$ & 0.631 \\
\hline Albumin of Fluid $(\mathrm{g} / \mathrm{dl})$ & $2.80 \pm 0.57$ & $2.85 \pm 0.57$ & $2.34 \pm 0.37$ & 0.062 \\
\hline $\mathrm{CRP}(\mathrm{mg} / \mathrm{L})$ & $7.49(1-30)$ & $6.3(1-30)$ & $19(14-25)$ & $<0.001$ \\
\hline $\mathrm{CRP} /$ albumin ratio & $2.17(0.24-12.02)$ & $1.9(0.24-10.38)$ & $4.27(3.87-12.02)$ & $<0.001$ \\
\hline $\mathrm{CRP} /$ Albumin of Fluid ratio & $2.68(0.28-13.59)$ & $2.5(0.28-12.22)$ & $7(6.25-13.59)$ & $<0.001$ \\
\hline
\end{tabular}

CRP: C-Reactive Protein

CRP value was found to be more statistically significant in group 2 patients $(\mathrm{p}<0.001)$ (Table 2$)$. Similarly, CRP/ albumin ratio and $\mathrm{CRP} / \mathrm{pf}$-Albumin ratio were found to be numerically and statistically more significant in group
2 patients ( $\mathrm{p}<0.001$ for both) (Table 2). Five patients had a lung cancer diagnosis, one had breast cancer, one had malignant neoplasm, one had larynx cancer, and five had chronic renal failure (Table 3).

\section{Table 3. Etiology of pericardial effusion}

\begin{tabular}{|l|c|c|c|}
\hline Cause & Discharged patients $(\mathbf{n}=49)$ & In hospital death $(\mathbf{n}=5)$ & Total $(\mathbf{n}=54)$ \\
\hline Breast CA & 1 & 0 & 1 \\
\hline Lung CA & 4 & 1 & 5 \\
\hline Larync CA & 1 & 0 & 1 \\
\hline Malignant neoplasm & 1 & 0 & 1 \\
\hline Chronic renal failure & 4 & 1 & 5 \\
\hline Idiopathic & 38 & 3 & 41 \\
\hline
\end{tabular}

\section{CA: Cancer}

Table 4 shows the association between the in-hospital mortality and CRP (odds ratio [OR]: 0.821, P: 0.004, 95.0\% confidence interval [CI]: 0.918-1.049), CRP/albumin ratio
(OR: 0.600, P: 0.011, 95.0\% CI: 0.406-0.888) and CRP/pfAlbumin ratio (OR: 0.608, P: 0.004, 95.0\% CI: 0.431-0.856) in patients who underwent pericardiocentesis. 
Table 4. Univariate regression analysis to determine in-hospital mortality

\begin{tabular}{|l|c|c|c|}
\hline & \multicolumn{3}{|c|}{ Univariate } \\
\hline Variables & $\mathrm{P}$ & OR & $95 \%$ CI \\
\hline Age (years) & 0.577 & 0.981 & $0.918-1.049$ \\
\hline Cancer & 0.733 & 1.500 & $0.146-15.461$ \\
\hline LDH (U/L) & 0.633 & 0.999 & $0.995-1.003$ \\
\hline LDH of Fluid (U/L) & 0.552 & 1.001 & $0.998-1.003$ \\
\hline Albumin (g/dl) & 0.625 & 1.540 & $0.273-8.679$ \\
\hline Albumin of Fluid (g/dl) & 0.078 & 4.531 & $0.843-24.353$ \\
\hline CRP (mg/L) & 0.004 & 0.821 & $0.716-0.940$ \\
\hline CRP/albumin ratio & 0.011 & 0.600 & $0.406-0.888$ \\
\hline CRP/Albumin of Fluid ratio & 0.004 & 0.608 & $0.431-0.856$ \\
\hline
\end{tabular}

CI: Confidence interval, OR: Odds ratio, Abbreviations in Table 1

Receiver operating characteristic (ROC) curve analysis indicates that a CRP/albumin ratio higher than 3.8 may predict in-hospital mortality. [ $\mathrm{p}<0.001)(100 \%$ sensitivity and $90 \%$ specificity, 0.939 area under the curve $95 \% \mathrm{CI}$ : 0.872-1.005)]. Similarly, it was observed that CRP/pfAlbumin ratio might predict in-hospital mortality when it is higher than the optimal cutoff value 6. [( $<<0.001)(100 \%$ sensitivity and $92 \%$ specificity, 0.947 area under the curve 95\% CI: 0.887-1.007)] (Figure 1).

Figure 1. Receiver operator characteristic curve of C-reactive protein/albumin and C-reactive protein/ pericardial fluid Albumin ratio to predict mortality in patients undergoing pericardiocentesis

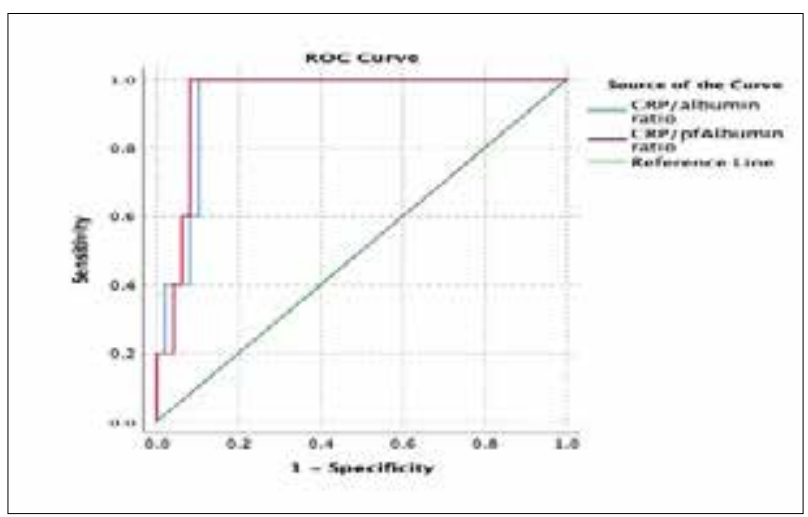

\section{DISCUSSION}

Pericardiocentesis is a valuable treatment procedure for diagnosing and treating effusions causing tamponade or serious effusions regardless of the etiology. Since the first time, it was described increasing experiences and using fluoroscopic and echocardiographic devices more frequently have increased the safety and success of the procedure. $^{11}$

As there is no randomized trial on pericardiocentesis, data on prognosis is limited. As far as is known, the prognosis is excellent in the absence of neoplastic disease and in cases where viral pathogens are implicated in the etiology. ${ }^{12}$ In our study, eight patients had malignancy with no association being observed with mortality in the short term. As can be seen, since the clinical parameters are not always sufficient, additional parameters are needed. Determining and using laboratory parameters in addition to clinical parameters for the prognosis of pericardiocentesis patients will be effective in predicting prognosis.

Several studies investigated and proved the usability of increased CRP levels. Being an acute phase reactant, it reaches plasma peak levels within 48 hours after being released from the liver. ${ }^{13,14}$ While high CRP levels were shown to be associated with metabolic disorder, and 
another study has associated the high CRP levels with poor negative outcomes in coronary artery disease. ${ }^{15}$ Additionally, they were found to be associated with mortality in patients with heart failure. ${ }^{16}$ Similar to the literature, increased CRP levels were observed to be associated with short-term in-hospital mortality in patients who underwent pericardiocentesis in our study.

Similar to CRP, another useful parameter is albumin, and recent studies have associated low $\mathrm{CRP} /$ albumin ratios with poor prognosis in patients with heart failure and cancer. ${ }^{17,18}$ In a long-term follow-up study such as 5 years, in which 212 pancreatic cancer patients were examined, it was shown that CRP values could be used for survival. In another meta-analysis, pre-treatment CRP/albumin ratio is a prognostic marker of poor overall survival (OS) and CSS in patients with gastric cancer (GC). In addition, high levels of CRP/albumin ratio are associated with clinicopathological features reflecting tumor progression. ${ }^{19,20}$ Regardless of the underlying specific etiology, CRP/albumin ratio was a decisive parameter in predicting the in-hospital mortality in patients who underwent pericardiocentesis in our study. Additionally, in the comparison of ROC curves, $\mathrm{CRP} / \mathrm{pf}$-albumin ratio was a more specific parameter than CRP/albumin ratio in predicting in-hospital mortality.

Moreover, studies have shown that serum LDH levels are a strong indicator of mortality in cancer patients. ${ }^{21}$ In our study, contrary to the literature, serum, and pericardial fluid LDH and albumin levels were not useful in predicting in-hospital mortality in patients who underwent pericardiocentesis. That differece might have been caused by the small number of patients and mixed study groups in our study.

Our study had some limitations. Firstly, it was a singlecenter study with a small number of patients. Secondly, as it was a retrospective study, we do not have information on the long-term usability of the results obtained in patients discharged with recovery. Prospective and multicenter studies are needed to use the study results globally.

For the first time in the literature, we demonstrated that $\mathrm{CRP} /$ albumin and CRP/pf-Albumin ratios are associated with in-hospital mortalities after pericardiocentesis, a life-saving procedure in severe pericardial effusions. We believe that this laboratory test which is easy to calculate, may be useful in the risk classification of the patients who underwent pericardiocentesis.

\section{Declarations}

The authors received no financial support for the research and/or authorship of this article. There is no conflict of interest.

This study was approved by the Çanakkale Onsekiz Mart University ethics committee of the hospital in which it took place in line with the recommendations of the Declaration of Helsinki (Date: 09.12.2020 Decision no: 2020-14).

\section{REFERENCES}

1. Adler $Y$, Charron P, Imazio M, Badano L, Barón-Esquivias G, Bogaert J, et al. 2015 ESC Guidelines for the diagnosis and management of pericardial diseases: The Task Force for the Diagnosis and Management of Pericardial Diseases of the European Society of Cardiology (ESC) Endorsed by: The European Association for Cardio-Thoracic Surgery (EACTS). Eur Heart J. 2015;36(42):2921-2964.

2. Roy CL, Minor MA, Brookhart MA, Choudhry NK. Does this patient with a pericardial effusion have cardiac tamponade? JAMA. 2007; 297(16):1810-1818.

3. Albugami S, Al-Husayni F, AlMalki A, Dumyati M, Zakri Y, AlRahimi J. Etiology of Pericardial Effusion and Outcomes Post Pericardiocentesis in the Western Region of Saudi Arabia: A Singlecenter Experience. Cureus. 2020;12(1):e6627.

4. Michalska-Kasiczak M, Bielecka-Dabrowa A, von Haehling S, Anker SD, Rysz J, Banach M. Biomarkers, myocardial fibrosis and co-morbidities in heart failure with preserved ejection fraction: an overview. Arch Med Sci. 2018;14(4):890-909.

5. Wang W, Ren D, Wang CS, Li T, Yao HC. High sensitivity C-reactive protein to prealbumin ratio measurement as a marker of the prognosis in acute coronary syndrome. Sci Rep. 2019;9(1):11583.

6. Yu B, Yang $\mathrm{P}, \mathrm{Xu} X$, Shao L. C-reactive protein for predicting all-cause mortality in patients with acute ischemic stroke: a meta-analysis. Biosci Rep. 2019;39(2):BSR20181135.

7. Utariani A, Rahardjo E, Perdanakusuma DS. Effects of Albumin Infusion on Serum Levels of Albumin, Proinflammatory Cytokines (TNF- $\alpha$, IL-1, and IL-6), CRP, and MMP-8; Tissue Expression of EGRF, ERK1, ERK2, TGF- $\beta$, Collagen, and MMP-8; and Wound Healing in Sprague Dawley Rats. Int J Inflam. 2020;2020:3254017.

8. Wang R, He M, Ou X, Xie X, Kang Y. CRP Albumin ratio is positively associated with poor outcome in patients with traumatic brain injury. Clin Neurol Neurosurg. 2020;195:106051.

9. Maisch B, Ristić AD, Seferović PM, Tsang TS. Interventional Pericardiology: Pericardiocentesis, Pericardioscopy, Pericardial Biopsy, Balloon Pericardiotomy and Intrapericardial Therapy. $1^{\text {st }} \mathrm{ed}$. Heidelberg: Springer Medizin Verlag, 2011.

10. Lang RM, Badano LP, Mor-Avi V, Afilalo J, Armstrong A, Ernande $\mathrm{L}$, et al. Recommendations for cardiac chamber quantification by echocardiography in adults: An update from the American Society of Echocardiography and the European Association of Cardiovascular Imaging. J Am Soc Echocardiogr. 2015;28(1):1-39.e14.

11. Krikorian JG, \& Mancock EW. Pericardiocentesis. Am J Med, 1978;65(5):808-14.

12. Jeong TD, Jang S, Park CJ, Chi HS. Prognostic relevance of pericardial effusion in patients with malignant diseases. Korean J Hematol. 2012;47(3):237-238. 
13. Rhodes B, Fürnrohr BG, Vyse TJ. C-reactive protein in rheumatology: biology and genetics. Nat Rev Rheumatol. 2011;7(5):282-289.

14. Sproston NR, Ashworth JJ. Role of C-Reactive Protein at Sites of Inflammation and Infection. Front Immunol. 2018;9:754.

15. Reilly SM, Saltiel AR. Adapting to obesity with adipose tissue inflammation. Nat Rev Endocrinol. 2017;13(11):633-43.

16. Rauchhaus M, Doehner W, Francis DP, Davos C, Kemp M, Liebenthal $\mathrm{C}$, et al. Plasma cytokine parameters and mortality in patients with chronic heart failure. Circulation 2000;102(25):3060-3067.

17. Gotsman I, Shauer A, Zwas DR, Tahiroglu I, Lotan C, Keren A. Low serum albumin: A significant predictor of reduced survival in patients with chronic heart failure. Clin Cardiol. 2019;42(3):365-372.

18. Kudou K, Saeki H, Nakashima Y, Kamori T, Kawazoe T, Haruta Y, et al. C-reactive protein/albumin ratio is a poor prognostic factor of esophagogastric junction and upper gastric cancer. J Gastroenterol Hepatol. 2019;34(2):355-363.

19. Nurmi AM, Mustonen HK, Stenman UH, Seppänen HE, Haglund CH. Combining CRP and CA19-9 in a novel prognostic score in pancreatic ductal adenocarcinoma. Sci Rep. 2021;11(1):781.

20. Yu J, Liu H, Zeng X, Zhao Y, Jiang D, Lu H, et al. Prognostic and clinicopathological significance of $\mathrm{C}$-reactive protein/albumin ratio (CAR) in patients with gastric cancer: A meta-analysis. PLoS One. 2021;16(4):e0250295.

21. Faloppi L, Del Prete M, Casadei Gardini A, Santini D, Silvestris N, Bianconi $\mathrm{M}$, et al. The correlation between LDH serum levels and clinical outcome in advanced biliary tract cancer patients treated with first line chemotherapy. Sci Rep. 2016;6:24136. 